Classification

Physics Abstracts

$05.90-61.40-64.75-82.70$

\title{
Swelling of an isolated polymer chain in a solvent
}

\section{J. Des Cloizeaux}

Service de Physique Théorique,

\author{
R. Conte
}

Service de Physique du Solide et de Résonance Magnétique,

and G. Jannink

Laboratoire Léon Brillouin (CEA-CNRS),

CEN-Saclay, 91191 Gif-sur-Yvette Cedex, France

(Reçu le 12 mars 1985, accepté le 15 mai 1985)

\begin{abstract}
Résumé. - Dans le cadre du modèle continu, on donne une expression précise du gonflement d'un polymère isolé en solution, pour toute valeur de l'interaction. La série de perturbation obtenue par Muthukumar et Nickel (1984) constitue la donnée initiale et une "méthode de renormalisation directe " est utilisée pour trouver le résultat final (à trois dimensions).
\end{abstract}

\begin{abstract}
In the framework of the continuous model, a precise expression of the swelling of an isolated polymer in solution is given for all values of the interaction. The perturbation series obtained by Muthukumar and Nickel (1984) constitutes the initial data and a " direct renormalization method " is used to get the final result (in three dimensions).
\end{abstract}

\section{Introduction.}

A polymer in a solution is currently represented as a Brownian chain with a $\delta$ interaction(Edwards' model). Let $R^{2}$ be the mean square end-to-end distance of the interacting chain and ${ }^{\circ} R^{2}$ the mean square end-to-end distance of the corresponding unperturbed Brownian chain. We may define the swelling of an isolated chain by the ratio :

$$
R^{2} /{ }^{\circ} R^{2}=\mathfrak{X}_{0}(z)
$$

where $\mathfrak{X}_{0}(z)$ is a function of the standard dimensionless parameter $z$ which determines the strength of the interaction. The function $\mathfrak{X}_{0}(z)$ can be expanded in powers of $z$ and the first terms of the expansion have been found a long time ago [1, 2]. Recently, more terms have been calculated in space dimensions $d=2$ and $d=3$ by M. Muthukumar and B. G. Nickel [3] who used known series expansions of Green's functions of the Landau-Ginzburg model ( $\varphi^{4}$ interaction). These series had been calculated by B. G. Nickel on a computer. In principle, by using the de Gennes transformation, they can be transformed into series representing the expansion of partition functions with respect to polymers. Finally $\mathfrak{X}_{0}(z)$ is given by the ratio of two partition functions. A 
difficulty studied by J. des Cloizeaux [4] and independently by M. Muthukumar and B. G. Nickel [3] comes from the appearance of spurious singularities in the partition functions. However these singularities do not appear in the swelling and, finally, Muthukumar and Nickel [3] found the result for $d=3$

$$
\begin{array}{r}
\mathscr{X}_{0}(z)=\left[1+\frac{4}{3} z-2.075385396 z^{2}+6.296879676 z^{3}-25.05725072 z^{4}+\right. \\
\left.+116.134785 z^{5}-594.71663 z^{6}+\cdots\right]
\end{array}
$$

for $d=2$

$$
X_{0}(z)=\left[1+\frac{z}{2}-0.12154525 z^{2}+0.02663136 z^{3}-0.13223603 z^{4}+\cdots\right] .
$$

These series contain much information but cannot be directly used because they are strongly divergent. However, for $d=3$, we have been able to extract from equation (1) the value of $\mathfrak{X}_{0}(z)$ for all values of $z$ by using a direct renormalization method; this method is very similar to the direct renormalization method introduced in 1980 by one of the authors $[5,6]$, and can be described as follows.

For any value of $d=4-\varepsilon$, we define the effective index $\sigma(z)$ by setting :

$$
\sigma(z)=\frac{\varepsilon}{2} z \frac{\partial}{\partial z} \ln \mathfrak{X}_{0}(z)
$$

Here the dimensionless parameter $z$ is given by $z=b S^{\varepsilon / 2}(2 \pi)^{-d / 2}$ where $b$ is the interaction and $S$ the "Brownian area " of the continuous chain [6] i.e. a quantity which is proportional to the number of links $N$ of the corresponding discrete chain. Thus we may also write :

$$
\sigma(z)=\left.S \frac{\partial}{\partial S} \ln \mathfrak{X}_{0}(z)\right|_{b=\text { const. }}
$$

and since $\mathfrak{X}_{0}$ is proportional to $N^{2 v-1}$ for $N \gg 1$, we see that :

$$
\lim _{z \rightarrow \infty} \sigma(z)=\sigma^{*}=2 v-1 .
$$

As $\sigma(0)=0$, we see that $\sigma(z)$ varies rather slowly with $z$. The effective index $\sigma$ can be considered as a quantity which has a deeper meaning than $z$ and we shall use it as the basic parameter of the problem (in Refs. [5, 6], the virial parameter $g$ was used instead of $\sigma$ ). We note that $\sigma(z)$ is given by a series expansion :

$$
\sigma(z)=a_{0} z+a_{1} z^{2}+\cdots
$$

and for $d=3$ and $d=2$ the first terms of this series are given by equations (1) and (2). Conversely, we may define a function $z[\sigma]$ and by inverting (5), we find :

$$
z[\sigma]=\frac{\sigma}{a_{0}}-\frac{a_{1}}{a_{0}^{2}} \sigma^{2}+\cdots .
$$

In the same way, we may reexpress the swelling in terms of $\sigma$ by setting :

$$
X_{0}[\sigma]=X_{0}(z[\sigma]) \text {. }
$$


Let us now introduce the quantity :

$$
w(z)=\varepsilon z \frac{\partial}{\partial z} \sigma(z)
$$

which might also be considered as a function of $\sigma$, by setting :

$$
w[\sigma]=w(z[\sigma]) .
$$

We note that from (8), (5) and (6), we may extract an expansion of $w[\sigma]$ of the form :

$$
w[\sigma]=b_{1} \sigma+b_{2} \sigma^{2}+\cdots=\varepsilon \sigma+\cdots .
$$

We also note that $w(z)$, which is the derivative of $\sigma(z)$ with respect to $\ln z$, must vanish when $z \rightarrow \infty$. As $\sigma \rightarrow \sigma^{*}$, when $z \rightarrow \infty$, we have :

$$
w\left[\sigma^{*}\right]=0 .
$$

The series (10) can be used to calculate $\sigma^{*}$ and the problem is to deduce from it an approximate expression of $w[\sigma]$ in the range :

$$
0<\sigma<\sigma^{*} .
$$

Now $w[\sigma]$ can be used to calculate $X_{0}[\sigma]$ and $z[\sigma]$. Thus equations (8) and (3) can be rewritten as follows :

$$
\begin{aligned}
\frac{1}{\varepsilon z} \frac{\mathrm{d} z}{\mathrm{~d} \sigma} & =\frac{1}{w[\sigma]} \\
\frac{\mathrm{d}}{\mathrm{d} \sigma} \ln X_{0} & =2 \frac{\sigma}{w[\sigma]} .
\end{aligned}
$$

By integrating and using equations (5) and (10), for $z \rightarrow 0$, we find the parametric representation :

$$
\begin{gathered}
z=\frac{\sigma}{a_{0}} \exp \left[\int_{0}^{\sigma} \mathrm{d} \sigma^{\prime}\left[\frac{\varepsilon}{w\left[\sigma^{\prime}\right]}-\frac{1}{\sigma^{\prime}}\right]\right] \\
x_{0}=\exp \left[2 \int_{0}^{\sigma} \mathrm{d} \sigma^{\prime} \frac{\sigma^{\prime}}{w\left[\sigma^{\prime}\right]}\right] .
\end{gathered}
$$

Let us now explain how the method works for $d=3(\varepsilon=1)$. The coefficients $b_{1}, b_{2}, \ldots, b_{6}$ which appear in the series (10) have been calculated by starting from (1). The result is :

$$
\begin{array}{lll}
b_{1}=1 & b_{3}=10.8060 & b_{5}=112.918 \\
b_{2}=-6.66962 & b_{4}=-39.2666 & b_{6}=-556.248 .
\end{array}
$$

We might estimate $w[\sigma]$ by keeping only the first terms in the series but this does not seem to be a very good approximation. On the other hand, it appears that the ratio $\left|\frac{b_{j}+1}{b_{j}}\right|$ does not increase rapidly with $j$. For this reason, we choose to approximate the unknown terms by a geometric series :

$$
w[\sigma]=b_{1} \sigma+\cdots+b_{4} \sigma^{4}+\frac{b_{5} \sigma^{5}}{1-b_{6} \sigma / b_{5}} .
$$

In other terms, we represent $w[\sigma]$ by its Padé approximant of type [5/1]. 
Thus for $\frac{1}{w[\sigma]}$ and $\frac{\sigma}{w[\sigma]}$, we obtain the approximate form :

$$
\begin{aligned}
& \frac{1}{w[\sigma]}=\frac{1}{\sigma}+\frac{A^{*}}{\sigma-\sigma^{*}}+\frac{A_{1}}{\sigma-\sigma_{1}}+\frac{A^{\prime}+i A^{\prime \prime}}{\sigma-\sigma^{\prime}-i \sigma^{\prime \prime}}+\frac{A^{\prime}-i A^{\prime \prime}}{\sigma-\sigma^{\prime}+i \sigma^{\prime \prime}} \\
& \frac{\sigma}{w[\sigma]}=\frac{B^{*}}{\sigma-\sigma^{*}}+\frac{B_{1}}{\sigma-\sigma_{1}}+\frac{B^{\prime}+i B^{\prime \prime}}{\sigma-\sigma^{\prime}-i \sigma^{\prime \prime}}+\frac{B^{\prime}-i B^{\prime \prime}}{\sigma-\sigma^{\prime}+i \sigma^{\prime \prime}} .
\end{aligned}
$$

Using these expressions, we find :

$$
\begin{aligned}
& z=\frac{3}{2} \sigma\left[1-\frac{\sigma}{\sigma^{*}}\right]^{A^{*}}\left[1-\frac{\sigma}{\sigma_{1}}\right]^{A_{1}}\left[1-\frac{2 \sigma \sigma^{\prime}-\sigma^{2}}{\sigma^{\prime 2}+\sigma^{\prime \prime 2}}\right]^{A^{\prime}} \exp \left[-2 A^{\prime \prime} \operatorname{Arctg}\left(\frac{\sigma \sigma^{\prime \prime}}{\sigma^{\prime 2}+\sigma^{\prime 2}-\sigma \sigma^{\prime}}\right)\right] \\
& \left(x_{0}\right)^{1 / 2}=\left[1-\frac{\sigma}{\sigma^{*}}\right]^{B^{*}}\left[1-\frac{\sigma}{\sigma_{1}}\right]^{B_{1}}\left[1-\frac{2 \sigma \sigma^{\prime}-\sigma^{2}}{\sigma^{\prime 2}+\sigma^{\prime \prime 2}}\right]^{B^{\prime}} \exp \left[-2 B^{\prime \prime} \operatorname{Arctg}\left(\frac{\sigma \sigma^{\prime \prime}}{\sigma^{\prime 2}+\sigma^{\prime 2}-\sigma \sigma^{\prime}}\right)\right]
\end{aligned}
$$

(where $B^{*}=\sigma^{*} A^{*}$ ).

For large $z$, we find :

$$
\ln X_{0}=\frac{2 A^{*}}{B^{*}} \ln z+C+\mathcal{O}\left(z^{1 / A^{*}}\right)
$$

(where $C$ is a constant).

The indices $v$ and $\Delta_{1}=\omega v$ are given by [6] :

$$
\begin{gathered}
v=\frac{1+\sigma^{*}}{2}=\frac{1}{2}+\frac{B^{*}}{2 A^{*}} \\
\Delta_{1}=-\frac{1}{2 A^{*}} .
\end{gathered}
$$

The numerical values are :

$$
\begin{aligned}
& \sigma^{*}=0.176903 \quad A^{*}=-1.056145 \quad B^{*}=-0.186836 \\
& \sigma_{1}=-0.218430 \quad A_{1}=0.026275 \quad B_{1}=-0.005739 \\
& \sigma^{\prime}=0.107488 \quad A^{\prime}=0.014935 \quad B^{\prime}=0.096287 \\
& \sigma^{\prime \prime}=0.556660 \quad A^{\prime \prime}=-0.170090 \quad B^{\prime \prime}=-0.009969 \text {. }
\end{aligned}
$$

These results for $d=3$ seem quite reasonable in particular, for large $z$, we find :

$$
\ln \mathfrak{X}_{0}=0.3538 \ln z+0.4278+\mathcal{O}\left(z^{-0.9468}\right) \text {. }
$$

For $v$ and $\Delta_{1}$, we obtain the values :

$$
v=0.588 \quad \Delta_{1}=0.473
$$

in complete agreement with the results of Le Guillou and Zinn-Justin [7].

The convergence of the process can be tested by computing successive estimates of $\sigma^{*}$ obtained by keeping only the first $n$ terms $(n \leqslant 6)$ in the series $(1)$. The calculation has been done by representing $w[\sigma]$, either by a Padé of type $[n-1 / 1]$ as in (13) or by a Padé $[n / 0]$ i.e. the truncated series itself. 
The results appear on the following table :

\begin{tabular}{|l|c|c|c|c|c|c|}
\hline & $n$ & 2 & 3 & 4 & 5 & 6 \\
\hline \multirow{2}{*}{ Padé $[(n-1) / 1]$} & $\sigma^{*}$ & & 0.1980 & 0.1823 & 0.1797 & 0.1769 \\
\cline { 2 - 7 } & $v$ & & 0.5990 & 0.5912 & 0.5899 & 0.5885 \\
\hline \multirow{2}{*}{ Padé $[n / \sigma]$} & $\sigma^{*}$ & 0.1499 & 0.2567 & 0.1677 & 0.1902 & 0.1701 \\
\cline { 2 - 7 } & $v$ & 0.5750 & 0.6283 & 0.5839 & 0.5951 & 0.5851 \\
\hline
\end{tabular}

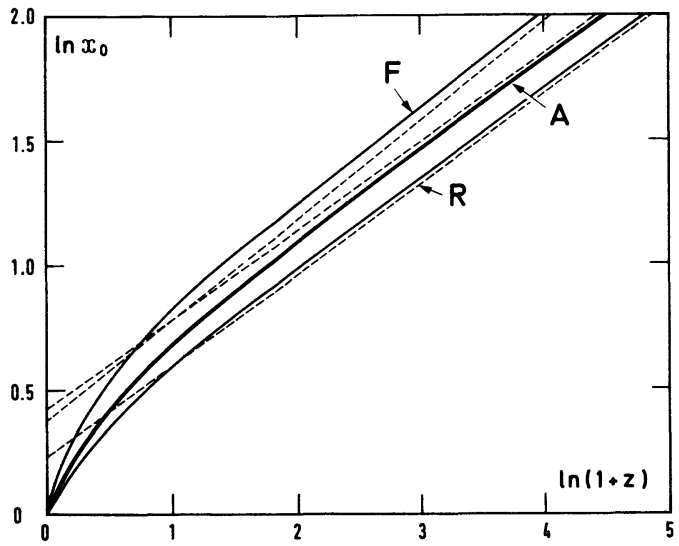

Fig. 1. - Plot of the swelling $\mathfrak{X}_{0}$ of a chain in three-dimensional space versus $\ln (1+z)$. A : Result given by the present calculation (asymptote $: \ln X_{0}=0.3538 \ln z+0.4278$ ). F $:$ Flory's formula (Eq. (16)) (asymptote $\left.: \ln \mathfrak{X}_{0}=0.4 \ln z+0.382\right)$. $R:$ A previous result obtained by second order $\varepsilon$-expansion and renormalization [6] (asymptote $: \ln \mathfrak{X}_{0}=0.367 \ln z+0.238$ ).

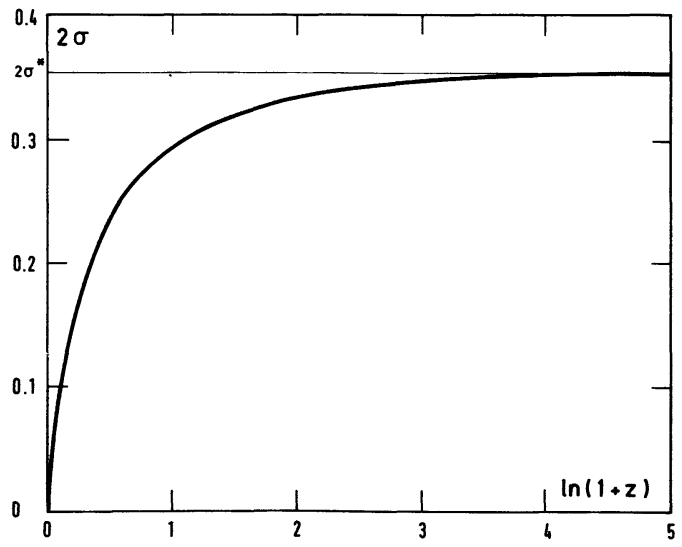

Fig. 2. - Plot of the effective index $2 \sigma$ versus $\ln (1+z)$ where $2 \sigma=\partial \ln x_{0} / \partial \ln z$ (case A of Fig. 1). (Note that for long polystyrene molecules in good solvents $0.1<\ln (1+z)<3.5$.) 
On figure $1, \mathfrak{X}_{0}$ is plotted versus $\ln (1+z)$. The result is compared with Flory's formula [8] :

$$
\left(X_{0}\right)^{5 / 2}-\left(X_{0}\right)^{3 / 2}=\frac{(3)^{3 / 2}}{2} z
$$

and an approximate expression obtained from a second order $\varepsilon$-expansion by direct renormalization [6]. Our result (14) is in agreement with all expectations and certainly gives precise values of the swelling for all values of $z$. On figure 2, $2 \sigma$ is plotted versus $\ln (1+z)$.

We also tried to apply the same method for $d=2$ but unsuccessfully : equation (2) does not contain as many terms as equation (1) and moreover the values of the terms which appear in equation (2) seem rather erratic. For these reasons, we shall not present here the results of the case $d=2$.

\section{References}

[1] Yamakawa, H., Modern theory of Polymer solutions (Harper and Row) 1971.

[2] Fixman, M., J. Chem. Phys. 23 (1955) 1656.

[3] Muthukumar, M., Nickel, B. G., J. Chem. Phys. 80 (1984) 5839.

[4] Des Cloizeaux, J., J. Physique 43 (1982) 1743.

[5] Des Cloizeaux, J., J. Physique Lett. 41 (1980) L-151.

[6] Des Cloizeaux, J., J. Physique 42 (1981) 635.

[7] Le Guillou, J. C. and Zinn-Justin, J., Phys. Rev. Lett. 39 (1977) 95.

[8] Flory, P. J., J. Chem. Phys. 17 (1949) 303.

FlORY, P. J. and Fox, T. G., J. Am. Chem. Soc. 73 (1951) 1904. 